\title{
Dynamics of Large Elongated RNA by NMR Carbon Relaxation
}

\author{
Alexandar L. Hansen and Hashim M. Al-Hashimi* \\ Department of Chemistry and Biophysics, \\ The University of Michigan, Ann Arbor, MI 48109, USA \\ Voice: (+1) 734-615-3361 \\ Fax: (+1) 734-647-4865 \\ E-mail: hashimi@umich.edu
}

\section{Supporting Information}

\section{Optimized Sampling Strategy}

In typical spin relaxation studies, a single decay will be measured using $\sim 10$ decay times, some of which are repeated to assess error in the measurement. Under reasonable conditions $\left(\sim 1 \mathrm{mM}{ }^{13} \mathrm{C} /{ }^{15} \mathrm{~N}\right.$ labeled RNA, 16 transients, 128 complex points, and recover delay of $1.5 \mathrm{sec}$ ), each 2D decay point can be measured in $\sim 2-3$ hours, resulting in a day of acquisition time per experiment $\left(\mathrm{R}_{1}\right.$ and $\left.\mathrm{R}_{1 \rho}\right)$. When sample conditions are not optimal and/or experiments need to be repeated under a variety of conditions/spin systems/spinlock field strengths, this can result in a prohibitively long amount of time. In situations where the relaxation decay is known to be mono-exponential, an optimized sampling strategy can be used, acquiring between two and five decay points, to dramatically reduce the experimental time. ${ }^{1,2}$

When relaxation times are very similar among all spin systems $\left(\mathrm{T}_{\max }<\sim 3 \mathrm{~T}_{\min }\right)$, only two decay points are necessary at $\mathrm{T}=0$ and $\mathrm{T}=\sim 1.2\left(\mathrm{~T}_{\max } \cdot \mathrm{T}_{\min }\right)^{1 / 2}$, the latter of which can be repeated several times. When times are not very similar $\left(\mathrm{T}_{\max }>\sim 3 \mathrm{~T}_{\min }\right)$, as can occur for $\mathrm{R} 2$ measurements in highly anisotropic RNAs, more complicated schemes can be used which use either three or four decay points. ${ }^{2}$ Obviously, an estimate for $\mathrm{T}_{\max }$ and $\mathrm{T}_{\min }$ is necessary to choose appropriate decay points and can be obtained from literature values of similar sized RNAs or from simulation. Alternatively, one can choose decay points experimentally by recording quick 1D traces of the relaxation experiment. A decay time of $\mathrm{T}=\sim 1.2\left(\mathrm{~T}_{\max } \cdot \mathrm{T}_{\min }\right)^{1 / 2}$ can be estimated as the decay time which results in a signal reduction of about $1 / 3$ and the longest time point, if necessary, should be chosen such that the signal remains 3-5 times the signal/noise ratio. Optimized sampling times in this study are presented in Table S2. 
a

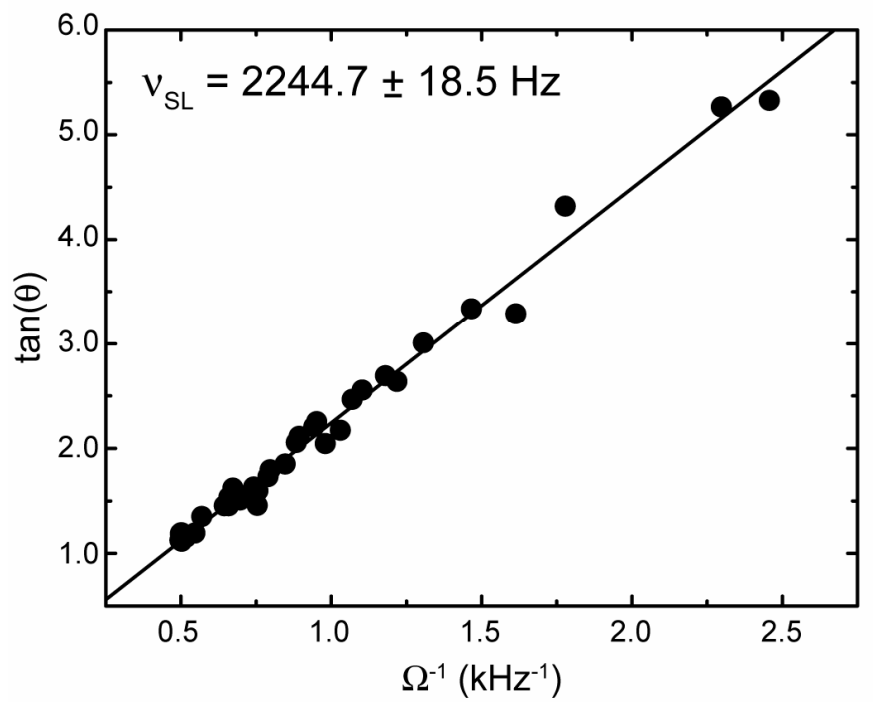

b

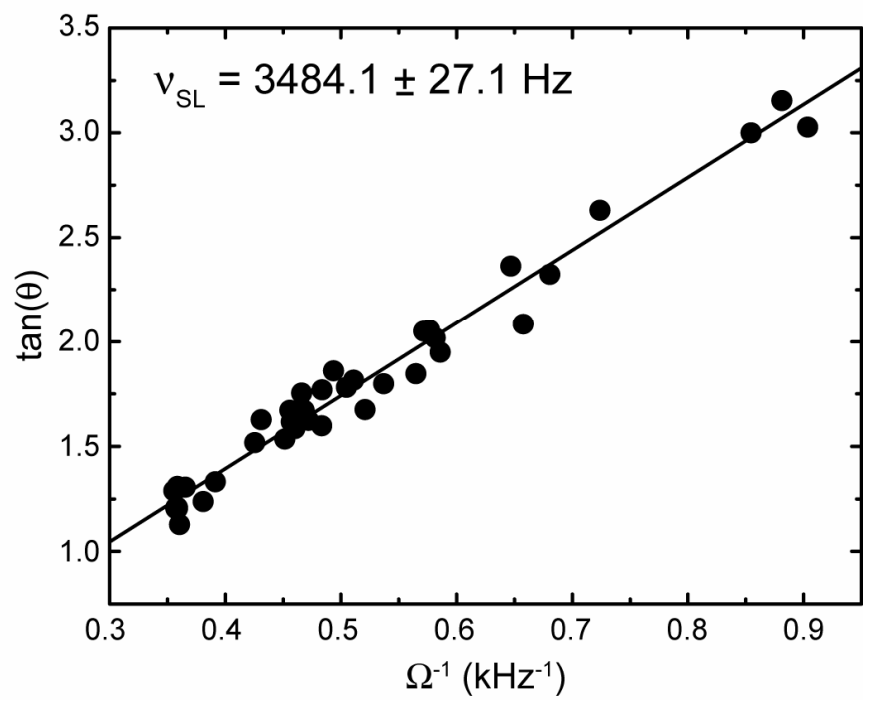

Figure S1. Calibration of spin lock used in the $R_{1 \rho}$ experiment for (a) C1' and (b) $\mathrm{C} 2 \mathrm{C} 5 \mathrm{C} 6$ and $\mathrm{C} 8$ carbons. 


\begin{tabular}{|c|c|c|c|c|c|c|c|c|c|c|c|}
\hline \multicolumn{12}{|c|}{ E-AU-TAR and E-AU-TAR+ARG } \\
\hline \multirow{2}{*}{ Spin } & \multicolumn{2}{|c|}{$\begin{array}{l}\text { On-Resonance } \\
\text { Inversion }\end{array}$} & \multicolumn{2}{|c|}{$\begin{array}{l}\text { On-Resonance } \\
\text { Refocusing }\end{array}$} & \multirow[b]{2}{*}{ Shape } & \multirow{2}{*}{$\begin{array}{l}\text { Off-Resonance } \\
\text { Inversion } \\
\text { Pulse width } \\
(\mu \mathrm{s})\end{array}$} & \multirow[b]{2}{*}{$\begin{array}{l}\text { Offset } \\
(\mathrm{ppm})\end{array}$} & \multirow{2}{*}{$\begin{array}{c}\text { Carrier } \\
\text { (ppm) }\end{array}$} & \multirow{2}{*}{$\begin{array}{l}\text { Sweep } \\
\text { Width } \\
\text { (ppm) }\end{array}$} & \multirow{2}{*}{$\begin{array}{l}\text { Spin Lock } \\
\text { Offset }(\mathrm{Hz})\end{array}$} & \multirow{2}{*}{$\begin{array}{r}\text { Spin Lock } \\
\text { power }(\mathrm{Hz})\end{array}$} \\
\hline & Shape & $\begin{array}{l}\text { Pulse width } \\
(\mu \mathrm{s})\end{array}$ & Shape & $\begin{array}{l}\text { Pulse Width } \\
\qquad(\mu \mathrm{s})\end{array}$ & & & & & & & \\
\hline C2 C6 C8 & ISNOB2 & 500 & REBURP & 1600 & ISNOB2 & 450 & 94 & 136.5 & 22 & +2750 & $\begin{array}{c}3484.1 \pm \\
27.1\end{array}$ \\
\hline $\mathrm{C} 5$ & ISNOB2 & 500 & REBURP & 1000 & IBURP2 & 750 & 152 & 98 & 14 & -2000 & $\begin{array}{c}3484.1 \pm \\
27.1\end{array}$ \\
\hline $\mathrm{C} 1^{\prime}$ & Q3 & 2000 & RSNOB & 1250 & Q3 & 2000 & 73 & 91 & 20 & +2000 & $\begin{array}{c}2244.7 \pm \\
18.5\end{array}$ \\
\hline \multicolumn{12}{|c|}{ E-GC-TAR, E-GC-TAR+ARG, and NE-TAR } \\
\hline $\mathrm{C} 2 \mathrm{C} 6 \mathrm{C} 8$ & Q3 & 750 & REBURP & 1000 & ISNOB2 & 500 & 99 & 142.5 & 27 & +1700 & $\begin{array}{c}3484.1 \pm \\
27.1\end{array}$ \\
\hline $\mathrm{C} 5$ & ISNOB2 & 450 & REBURP & 1000 & IBURP2 & 750 & 152 & 99 & 20 & -1750 & $\begin{array}{c}3484.1 \pm \\
27.1\end{array}$ \\
\hline $\mathrm{C} 1^{\prime}$ & Q3 & 2000 & RSNOB & 1250 & Q3 & 2000 & 73 & 91 & 20 & +2000 & $\begin{array}{c}2244.7 \pm \\
18.5 \\
\end{array}$ \\
\hline
\end{tabular}

Table S1. Parameters for the $R_{1}$ and $R_{1 \rho}$ experiments. A recycle delay of $1.5 \mathrm{sec}$ was used for all experiments. 


\begin{tabular}{|c|c|c|c|}
\hline \multirow[b]{2}{*}{$R_{1}$} & \multicolumn{3}{|c|}{ Delays (ms) } \\
\hline & $\mathrm{C} 2, \mathrm{C} 6$, and $\mathrm{C} 8$ & $\mathrm{C} 5$ & $\mathrm{C} 1^{\prime}$ \\
\hline \multicolumn{4}{|l|}{ TD E-TAR } \\
\hline $25^{\circ} \mathrm{C}$ & $20,60(\mathrm{x} 2), 100,200,400,700(\mathrm{x} 2), 1000$ & $20,760(x 4)$ & na \\
\hline $7^{\circ} \mathrm{C}$ & $20,700(x 4)$ & na & na \\
\hline non-selective & $20,60(\mathrm{x} 2), 100,200,400,700(\mathrm{x} 2), 1000$ & na & na \\
\hline $\begin{array}{l}\text { Argininamide bound } \\
\text { non-TD E-TAR }\end{array}$ & $20,860(x 4)$ & $20,760(x 4)$ & na \\
\hline $25^{\circ} \mathrm{C}$ & 20, 60 (x2), 100, 200, 400, 700 (x2), 1000 & na & $20,1000(x 4)$ \\
\hline $7^{\circ} \mathrm{C}$ & 20 & na & na \\
\hline $\begin{array}{l}\text { Argininamide bound } \\
\text { non-TD NE-TAR }\end{array}$ & na & na & $20,1000(x 4)$ \\
\hline $25^{\circ} \mathrm{C}$ & $20,580(\times 4)$ & $20,660(x 4)$ & $20,640(x 4)$ \\
\hline \multicolumn{4}{|l|}{$R_{1 \rho}$} \\
\hline \multicolumn{4}{|l|}{ TD E-TAR } \\
\hline $25^{\circ} \mathrm{C}$ & $4,8(x 2), 12,24,32,48(x 2), 64$ & 4, $23(x 4)$ & na \\
\hline $7^{\circ} \mathrm{C}$ & 4, $10(\mathrm{x} 2), 18(\mathrm{x} 2)$ & na & na \\
\hline $\begin{array}{l}\text { Argininamide bound } \\
\text { non-TD E-TAR }\end{array}$ & 4, 15 (x2), $30(\mathrm{x} 2)$ & $4,23(x 4)$ & na \\
\hline $25^{\circ} \mathrm{C}$ & $4,8(x 2), 12,24,32,48(x 2), 64$ & na & $4,12(x 2), 32(x 2)$ \\
\hline $7^{\circ} \mathrm{C}$ & 4 & na & na \\
\hline $\begin{array}{l}\text { Argininamide bound } \\
\text { non-TD NE-TAR }\end{array}$ & na & na & $4,12(x 2), 32(x 2)$ \\
\hline $25^{\circ} \mathrm{C}$ & $4,60(x 4)$ & $4,70(x 4)$ & $4,78(x 4)$ \\
\hline
\end{tabular}

Table S2. Relaxation delays used in the $R_{1}$ and $R_{1 p}$ experiments. Duplicate and quadruplicate measurements used to estimate error are indicated using "x2" and "x4", respectively.

\begin{tabular}{cccc}
\hline Residue & Carbon & $R_{1}(\mathrm{~Hz})$ & $R_{2}(\mathrm{~Hz})$ \\
\hline G17 & C8 & $1.929 \pm 0.003$ & $26.99 \pm 0.04$ \\
G18 & C1 ${ }^{\prime}$ & $1.458 \pm 0.017$ & $26.58 \pm 0.04$ \\
& C8 & $2.002 \pm 0.002$ & $27.66 \pm 0.04$ \\
C19 & C5 & $2.275 \pm 0.004$ & $30.37 \pm 0.03$ \\
& C6 & $2.642 \pm 0.002$ & $33.55 \pm 0.03$ \\
A20 & C1 $^{\prime}$ & $1.531 \pm 0.011$ & $21.97 \pm 0.05$ \\
& C2 & $2.140 \pm 0.006$ & $30.30 \pm 0.03$ \\
& C8 & $1.999 \pm 0.003$ & $27.58 \pm 0.04$ \\
G21 & C1 ${ }^{\prime}$ & $1.563 \pm 0.010$ & $21.25 \pm 0.05$ \\
& C8 & $1.936 \pm 0.005$ & $28.73 \pm 0.03$ \\
A22 & C1 ${ }^{\prime}$ & $1.650 \pm 0.009$ & $21.88 \pm 0.05$ \\
& C2 & $2.043 \pm 0.004$ & $32.54 \pm 0.03$ \\
& C8 & $1.949 \pm 0.004$ & $27.95 \pm 0.04$ \\
U23 & C1 ${ }^{\prime}$ & $2.010 \pm 0.009$ & $17.25 \pm 0.06$ \\
& C5 & $2.349 \pm 0.004$ & $23.94 \pm 0.04$ \\
& C6 & $2.735 \pm 0.004$ & $28.96 \pm 0.03$ \\
C24 & C5 & $2.541 \pm 0.004$ & $20.03 \pm 0.05$ \\
& C6 & $2.821 \pm 0.004$ & $23.12 \pm 0.04$ \\
U25 & C5 & $2.407 \pm 0.003$ & $21.22 \pm 0.05$ \\
& C6 & $2.762 \pm 0.003$ & $25.89 \pm 0.04$ \\
G26 & C8 & $1.901 \pm 0.002$ & $29.07 \pm 0.03$ \\
A27 & C1 ${ }^{\prime}$ & $1.401 \pm 0.010$ & $26.28 \pm 0.04$ \\
& C2 & $2.005 \pm 0.006$ & $32.85 \pm 0.03$ \\
& C8 & $1.937 \pm 0.003$ & $29.18 \pm 0.03$ \\
G28 & C1 ${ }^{\prime}$ & $1.478 \pm 0.009$ & $24.07 \pm 0.04$
\end{tabular}




\begin{tabular}{|c|c|c|c|}
\hline & $\mathrm{C} 8$ & $1.977 \pm 0.004$ & $28.15 \pm 0.04$ \\
\hline \multirow[t]{2}{*}{$\mathrm{C} 29$} & $\mathrm{C} 5$ & $2.280 \pm 0.008$ & $29.51 \pm 0.03$ \\
\hline & C6 & $2.550 \pm 0.005$ & $36.25 \pm 0.03$ \\
\hline \multirow{3}{*}{ U31 } & $\mathrm{C} 1^{\prime}$ & $1.576 \pm 0.010$ & $21.52 \pm 0.05$ \\
\hline & $\mathrm{C} 5$ & $2.273 \pm 0.004$ & $28.63 \pm 0.03$ \\
\hline & C6 & $2.526 \pm 0.005$ & $35.91 \pm 0.03$ \\
\hline \multirow[t]{3}{*}{$\mathrm{U} 32$} & $\mathrm{C} 1^{\prime}$ & $1.600 \pm 0.011$ & $20.27 \pm 0.05$ \\
\hline & $\mathrm{C} 5$ & $1.912 \pm 0.005$ & $21.34 \pm 0.05$ \\
\hline & C6 & $2.137 \pm 0.005$ & $24.11 \pm 0.04$ \\
\hline \multirow[t]{3}{*}{$\mathrm{C} 33$} & $\mathrm{C} 1^{\prime}$ & $1.564 \pm 0.014$ & $22.91 \pm 0.04$ \\
\hline & $\mathrm{C} 5$ & $2.164 \pm 0.007$ & $28.92 \pm 0.03$ \\
\hline & C6 & $2.326 \pm 0.003$ & $33.76 \pm 0.03$ \\
\hline \multirow[t]{2}{*}{ G34 } & $\mathrm{C} 1^{\prime}$ & $1.577 \pm 0.010$ & $20.95 \pm 0.05$ \\
\hline & $\mathrm{C} 8$ & $1.888 \pm 0.003$ & $25.25 \pm 0.04$ \\
\hline \multirow[t]{2}{*}{ G36 } & $\mathrm{C} 1^{\prime}$ & $1.440 \pm 0.012$ & $23.17 \pm 0.04$ \\
\hline & $\mathrm{C} 8$ & $1.972 \pm 0.003$ & $27.30 \pm 0.04$ \\
\hline \multirow[t]{3}{*}{ C37 } & $\mathrm{C} 1^{\prime}$ & $1.537 \pm 0.009$ & $22.69 \pm 0.04$ \\
\hline & $\mathrm{C} 5$ & $2.295 \pm 0.004$ & $29.59 \pm 0.03$ \\
\hline & C6 & $2.542 \pm 0.004$ & $36.27 \pm 0.03$ \\
\hline \multirow[t]{2}{*}{$\mathrm{U} 38$} & $\mathrm{C} 5$ & $2.253 \pm 0.007$ & $29.00 \pm 0.03$ \\
\hline & $\mathrm{C} 6$ & $2.546 \pm 0.004$ & $36.74 \pm 0.03$ \\
\hline \multirow[t]{2}{*}{ C39 } & $\mathrm{C} 5$ & $2.308 \pm 0.006$ & $29.77 \pm 0.03$ \\
\hline & $\mathrm{C} 6$ & $2.477 \pm 0.007$ & $36.86 \pm 0.03$ \\
\hline \multirow[t]{2}{*}{$\mathrm{U} 40$} & $\mathrm{C} 1^{\prime}$ & $1.665 \pm 0.010$ & $20.81 \pm 0.05$ \\
\hline & $\mathrm{C} 5$ & $2.243 \pm 0.006$ & $28.29 \pm 0.04$ \\
\hline \multirow[t]{3}{*}{$\mathrm{C} 41$} & $\mathrm{C} 1^{\prime}$ & $1.534 \pm 0.009$ & $23.08 \pm 0.04$ \\
\hline & C5 & $2.211 \pm 0.009$ & $28.98 \pm 0.03$ \\
\hline & $\mathrm{C} 6$ & $2.428 \pm 0.005$ & $35.97 \pm 0.03$ \\
\hline \multirow[t]{2}{*}{ U42 } & $\mathrm{C} 1^{\prime}$ & $1.561 \pm 0.011$ & $24.89 \pm 0.04$ \\
\hline & C5 & $2.168 \pm 0.011$ & $29.89 \pm 0.03$ \\
\hline \multirow[t]{2}{*}{ G43 } & $\mathrm{C} 1^{\prime}$ & $1.602 \pm 0.014$ & $22.20 \pm 0.05$ \\
\hline & $\mathrm{C} 8$ & $1.978 \pm 0.005$ & $28.11 \pm 0.04$ \\
\hline \multirow[t]{3}{*}{ C44 } & $\mathrm{C} 1^{\prime}$ & $1.669 \pm 0.012$ & $19.69 \pm 0.05$ \\
\hline & $\mathrm{C} 5$ & $2.275 \pm 0.007$ & $29.63 \pm 0.03$ \\
\hline & C6 & $2.621 \pm 0.005$ & $34.14 \pm 0.03$ \\
\hline \multirow[t]{3}{*}{$\mathrm{C} 45$} & $\mathrm{C} 1^{\prime}$ & $1.807 \pm 0.010$ & $17.06 \pm 0.06$ \\
\hline & $\mathrm{C} 5$ & $2.351 \pm 0.018$ & $27.28 \pm 0.04$ \\
\hline & $\mathrm{C} 6$ & $2.569 \pm 0.004$ & $32.70 \pm 0.03$ \\
\hline
\end{tabular}

Table S3. $R_{1}$ and $R_{2}$ measured for NE-TAR using standard (non-TROSY).

\begin{tabular}{ccccccc}
\hline Residue & Carbon & $R_{1}{ }^{\mathrm{a}}(\mathrm{Hz})$ & $R_{1}{ }^{\mathrm{b}}(\mathrm{Hz})$ & $\sigma_{\mathrm{C} 5 \mathrm{C} 6}(\mathrm{~Hz})$ & $R_{2}(\mathrm{~Hz})$ & $\mathrm{NOE}^{\mathrm{c}}$ \\
\hline $\mathrm{G} 17$ & $\mathrm{C} 8$ & $0.942 \pm 0.019$ & $0.909 \pm 0.040$ & & $104.64 \pm 2.28$ & $1.257 \pm 0.128$ \\
$\mathrm{G} 18$ & $\mathrm{C} 8$ & $0.980 \pm 0.016$ & $0.972 \pm 0.066$ & & $112.90 \pm 2.38$ & $1.218 \pm 0.121$ \\
$\mathrm{C} 19$ & $\mathrm{C}^{\mathrm{d}}$ & $1.631 \pm 0.045$ & & & $110.00 \pm 5.08$ & \\
& $\mathrm{C} 6$ & $1.600 \pm 0.056$ & $0.925 \pm 0.088$ & $-0.675 \pm 0.104$ & $130.58 \pm 8.69$ & \\
$\mathrm{~A} 20$ & $\mathrm{C} 1^{\prime}$ & $0.814 \pm 0.103$ & & $85.17 \pm 15.10$ & \\
& $\mathrm{C} 2$ & $1.086 \pm 0.024$ & & & $123.82 \pm 8.47$ & $1.147 \pm 0.146$ \\
& $\mathrm{C} 8$ & $1.069 \pm 0.027$ & & & $99.31 \pm 6.24$ & $1.252 \pm 0.154$ \\
$\mathrm{G} 21$ & $\mathrm{C} 1^{\prime}$ & $0.975 \pm 0.063$ & & & $90.63 \pm 8.52$ & \\
& $\mathrm{C} 8$ & $1.027 \pm 0.016$ & $1.079 \pm 0.033$ & & $107.28 \pm 2.15$ & $1.188 \pm 0.118$ \\
$\mathrm{~A} 22$ & $\mathrm{C} 1{ }^{\prime}$ & $1.192 \pm 0.063$ & & & $93.33 \pm 6.13$ & \\
& $\mathrm{C} 2$ & $1.143 \pm 0.017$ & & & $123.28 \pm 5.39$ & $1.174 \pm 0.119$ \\
& $\mathrm{C} 8$ & $1.190 \pm 0.025$ & & & $102.21 \pm 4.34$ & $1.243 \pm 0.127$
\end{tabular}




\begin{tabular}{|c|c|c|c|c|c|c|}
\hline \multirow[t]{3}{*}{ U23 } & $\mathrm{C} 1^{\prime}$ & $1.805 \pm 0.027$ & & & $51.97 \pm 1.14$ & \\
\hline & $C 5^{\mathrm{d}}$ & $2.044 \pm 0.028$ & & & $87.49 \pm 1.71$ & \\
\hline & C6 & $2.088 \pm 0.038$ & & & $107.63 \pm 4.51$ & \\
\hline \multirow[t]{2}{*}{$\mathrm{C} 24$} & $C 5^{\mathrm{d}}$ & $2.315 \pm 0.017$ & & & $55.52 \pm 0.54$ & \\
\hline & $\mathrm{C} 6$ & $2.310 \pm 0.016$ & $2.057 \pm 0.037$ & $-0.253 \pm 0.040$ & $67.09 \pm 0.53$ & \\
\hline \multirow[t]{3}{*}{$\mathrm{U} 25$} & $\mathrm{C} 1^{\prime}$ & $1.756 \pm 0.032$ & & & $62.29 \pm 1.70$ & \\
\hline & $\mathrm{C} 5^{\mathrm{d}}$ & $2.210 \pm 0.023$ & & & $70.12 \pm 1.07$ & \\
\hline & C6 & $2.366 \pm 0.022$ & & & $73.79 \pm 1.28$ & \\
\hline G26 & $\mathrm{C} 8$ & $1.341 \pm 0.013$ & $1.270 \pm 0.035$ & & $87.51 \pm 1.11$ & $1.220 \pm 0.077$ \\
\hline \multirow[t]{3}{*}{$\mathrm{A} 27$} & $\mathrm{C} 1^{\prime}$ & $1.208 \pm 0.078$ & & & $80.74 \pm 8.59$ & \\
\hline & $\mathrm{C} 2$ & $1.355 \pm 0.020$ & & & $101.98 \pm 3.59$ & $1.198 \pm 0.097$ \\
\hline & $\mathrm{C} 8$ & $1.364 \pm 0.021$ & & & $83.80 \pm 2.67$ & $1.214 \pm 0.085$ \\
\hline G28 & $\mathrm{C} 8$ & $1.470 \pm 0.015$ & $1.410 \pm 0.023$ & & $79.15 \pm 0.99$ & $1.210 \pm 0.065$ \\
\hline \multirow[t]{2}{*}{$\mathrm{C} 29$} & $C 5^{\mathrm{d}}$ & $2.102 \pm 0.033$ & & & $93.68 \pm 2.03$ & \\
\hline & $\mathrm{C} 6$ & $2.145 \pm 0.040$ & $1.877 \pm 0.066$ & $-0.268 \pm 0.078$ & $103.47 \pm 2.59$ & \\
\hline \multirow[t]{3}{*}{ U31 } & $\mathrm{C} 1^{\prime}$ & $1.369 \pm 0.045$ & & & $56.99 \pm 2.52$ & \\
\hline & $\mathrm{C} 5^{\mathrm{d}}$ & $2.153 \pm 0.040$ & & & $91.03 \pm 2.42$ & \\
\hline & C6 & $2.143 \pm 0.058$ & & & $99.70 \pm 6.06$ & \\
\hline \multirow[t]{3}{*}{ U32 } & $\mathrm{C} 1^{\prime}$ & $1.411 \pm 0.021$ & & & $54.16 \pm 1.35$ & \\
\hline & $C 5^{\mathrm{d}}$ & $1.977 \pm 0.025$ & & & $67.58 \pm 1.27$ & \\
\hline & $\mathrm{C} 6$ & $2.100 \pm 0.026$ & & & $61.91 \pm 1.39$ & \\
\hline \multirow[t]{3}{*}{$\mathrm{C} 33$} & $\mathrm{C} 1^{\prime}$ & $1.505 \pm 0.024$ & & & $56.74 \pm 1.68$ & \\
\hline & $C 5^{\mathrm{d}}$ & $2.017 \pm 0.043$ & & & $91.29 \pm 2.56$ & \\
\hline & $\mathrm{C} 6$ & $2.014 \pm 0.032$ & $1.538 \pm 0.040$ & $-0.476 \pm 0.052$ & $96.51 \pm 2.38$ & \\
\hline \multirow[t]{2}{*}{ G34 } & $\mathrm{C} 1^{\prime}$ & $1.435 \pm 0.019$ & & & $58.12 \pm 1.24$ & \\
\hline & $\mathrm{C} 8$ & $1.448 \pm 0.009$ & $1.381 \pm 0.021$ & & $67.53 \pm 0.55$ & $1.270 \pm 0.049$ \\
\hline \multirow[t]{2}{*}{ G36 } & $\mathrm{C} 1^{\prime}$ & $1.366 \pm 0.026$ & & & $62.00 \pm 1.99$ & \\
\hline & $\mathrm{C} 8$ & $1.456 \pm 0.011$ & $1.385 \pm 0.029$ & & $81.40 \pm 0.83$ & $1.259 \pm 0.070$ \\
\hline $\mathrm{C} 37$ & $C 5^{\mathrm{d}}$ & $2.098 \pm 0.032$ & & & $92.47 \pm 2.01$ & \\
\hline \multirow[t]{3}{*}{ U38 } & $\mathrm{C} 1^{\prime}$ & $1.424 \pm 0.043$ & & & $62.94 \pm 2.70$ & \\
\hline & $C 5^{\mathrm{d}}$ & $2.015 \pm 0.046$ & & & $92.65 \pm 2.95$ & \\
\hline & C6 & $2.199 \pm 0.064$ & & & $97.67 \pm 6.84$ & \\
\hline \multirow[t]{2}{*}{ C39 } & $\mathrm{C} 1^{\prime}$ & $1.367 \pm 0.025$ & & & $65.61 \pm 1.92$ & \\
\hline & $C 5^{\mathrm{d}}$ & $2.168 \pm 0.042$ & & & $95.14 \pm 2.50$ & \\
\hline \multirow[t]{2}{*}{$\mathrm{U} 40$} & $\mathrm{C} 1^{\prime}$ & $1.478 \pm 0.053$ & & & $67.47 \pm 3.62$ & \\
\hline & $C 5^{\mathrm{d}}$ & $2.055 \pm 0.046$ & & & $91.31 \pm 2.93$ & \\
\hline \multirow[t]{2}{*}{$\mathrm{C} 41$} & $C 5^{d}$ & $1.711 \pm 0.044$ & & & $111.28 \pm 4.51$ & \\
\hline & $\mathrm{C} 6$ & $1.672 \pm 0.048$ & $1.079 \pm 0.085$ & $-0.593 \pm 0.097$ & $140.71 \pm 6.47$ & \\
\hline \multirow[t]{2}{*}{ U42 } & $\mathrm{C} 1^{\prime}$ & $1.084 \pm 0.096$ & & & $73.88 \pm 14.08$ & \\
\hline & $C 5^{\mathrm{d}}$ & $1.690 \pm 0.058$ & & & $114.08 \pm 6.32$ & \\
\hline G43 & $\mathrm{C} 8$ & $0.994 \pm 0.020$ & $1.002 \pm 0.043$ & & $97.75 \pm 2.28$ & $1.265 \pm 0.140$ \\
\hline $\mathrm{C} 44$ & $C 5^{\mathrm{d}}$ & $1.671 \pm 0.047$ & & & $117.13 \pm 5.87$ & \\
\hline
\end{tabular}

Table S4. $R_{1}, R_{2}, \sigma_{\mathrm{C} 5 \mathrm{C} 6}$ and C-H NOE measured for E-TAR using the TROSY-detected (C2 C5 C6 C8) and non-TROSY detected (C1') experiments. Shown are the $R_{1}$ values measured ${ }^{\mathrm{a}}$ with and ${ }^{\mathrm{b}}$ without selective inversion of the carbon of interest during the INEPT. ${ }^{\mathrm{C}}$ Non-TROSY detected experiments were used to determine heteronuclear NOE values for C2 and C8 spins. ${ }^{\mathrm{d}}$ To minimize overlap with water signals, the F2 semi-TROSY peak $\left(\Omega_{\mathrm{C} 5}+\pi \mathrm{J}_{\mathrm{CH}}, \Omega_{\mathrm{H} 5}+\pi \mathrm{J}_{\mathrm{CH}}\right)$ was detected.

\begin{tabular}{ccccc}
\hline Residue & Atom & $R_{1}(\mathrm{~Hz})$ & $R_{2}(\mathrm{~Hz})$ & NOE $^{\mathrm{a}}$ \\
\hline G17 & C8 & $0.827 \pm 0.018$ & $116.65 \pm 3.83$ & $1.180 \pm 0.072$ \\
G18 & C8 & $0.924 \pm 0.017$ & $117.18 \pm 3.59$ & $1.187 \pm 0.084$ \\
C19 & C5 $^{\mathrm{b}}$ & $1.713 \pm 0.038$ & $123.67 \pm 4.22$ &
\end{tabular}




\begin{tabular}{|c|c|c|c|c|}
\hline \multirow{3}{*}{ A20 } & $\mathrm{C} 6$ & $1.517 \pm 0.049$ & $139.18 \pm 8.13$ & \\
\hline & $\mathrm{C} 2$ & $0.928 \pm 0.021$ & $138.04 \pm 7.92$ & $1.342 \pm 0.213$ \\
\hline & $\mathrm{C} 8$ & $0.914 \pm 0.023$ & $118.05 \pm 5.13$ & $1.238 \pm 0.184$ \\
\hline \multirow[t]{2}{*}{$\mathrm{G} 21$} & $\mathrm{C} 1^{\prime}$ & $0.883 \pm 0.060$ & $85.29 \pm 5.96$ & \\
\hline & $\mathrm{C} 8$ & $0.925 \pm 0.019$ & $111.01 \pm 3.57$ & $1.195 \pm 0.082$ \\
\hline \multirow[t]{2}{*}{ A22 } & $\mathrm{C} 2$ & $1.058 \pm 0.021$ & $122.11 \pm 6.15$ & $1.146 \pm 0.200$ \\
\hline & $\mathrm{C} 8$ & $1.012 \pm 0.029$ & $116.41 \pm 5.36$ & $1.093 \pm 0.185$ \\
\hline \multirow[t]{3}{*}{ U23 } & $\mathrm{C} 1^{\prime}$ & $1.356 \pm 0.083$ & $50.85 \pm 5.82$ & \\
\hline & $\mathrm{C} 5^{\mathrm{b}}$ & $1.736 \pm 0.067$ & $115.49 \pm 5.95$ & \\
\hline & C6 & $1.651 \pm 0.069$ & $101.21 \pm 10.78$ & \\
\hline \multirow{3}{*}{$\mathrm{C} 24$} & $\mathrm{C} 1^{\prime}$ & $2.115 \pm 0.003$ & $21.72 \pm 0.27$ & \\
\hline & $\mathrm{C} 5^{\mathrm{b}}$ & $2.323 \pm 0.005$ & $30.85 \pm 0.32$ & \\
\hline & $\mathrm{C} 6$ & $2.390 \pm 0.006$ & $35.63 \pm 0.39$ & \\
\hline \multirow{3}{*}{$\mathrm{U} 25$} & $\mathrm{C} 1^{\prime}$ & $1.935 \pm 0.012$ & $36.16 \pm 0.73$ & \\
\hline & $\mathrm{C} 5^{\mathrm{b}}$ & $2.176 \pm 0.011$ & $40.70 \pm 0.65$ & \\
\hline & $\mathrm{C} 6$ & $2.550 \pm 0.013$ & $38.92 \pm 0.48$ & \\
\hline G26 & $\mathrm{C} 8$ & $1.021 \pm 0.016$ & $111.24 \pm 2.99$ & $1.224 \pm 0.075$ \\
\hline \multirow[t]{3}{*}{$\mathrm{A} 27$} & $\mathrm{C} 1^{\prime}$ & $0.815 \pm 0.103$ & $46.37 \pm 14.74$ & \\
\hline & $\mathrm{C} 2$ & $0.999 \pm 0.021$ & $123.48 \pm 7.75$ & $1.163 \pm 0.182$ \\
\hline & $\mathrm{C} 8$ & $1.114 \pm 0.028$ & $111.44 \pm 4.88$ & $1.112 \pm 0.176$ \\
\hline G28 & $\mathrm{C} 8$ & $1.026 \pm 0.018$ & $107.98 \pm 3.27$ & $1.213 \pm 0.083$ \\
\hline \multirow[t]{2}{*}{$\mathrm{C} 29$} & $\mathrm{C} 5^{\mathrm{b}}$ & $1.833 \pm 0.038$ & $120.11 \pm 3.85$ & \\
\hline & $\mathrm{C} 6$ & $1.544 \pm 0.051$ & $132.28 \pm 7.93$ & \\
\hline \multirow[t]{3}{*}{ U31 } & $\mathrm{C} 1^{\prime}$ & $1.273 \pm 0.097$ & $55.45 \pm 8.89$ & \\
\hline & $\mathrm{C} 5^{\mathrm{b}}$ & $1.894 \pm 0.063$ & $118.69 \pm 5.34$ & \\
\hline & C6 & $1.775 \pm 0.052$ & $117.27 \pm 7.76$ & \\
\hline \multirow[t]{3}{*}{$\mathrm{U} 32$} & $\mathrm{C} 1^{\prime}$ & $1.182 \pm 0.038$ & $59.25 \pm 3.24$ & \\
\hline & $\mathrm{C} 5^{\mathrm{b}}$ & $1.698 \pm 0.029$ & $80.03 \pm 1.99$ & \\
\hline & C6 & $1.701 \pm 0.025$ & $77.42 \pm 2.04$ & \\
\hline \multirow[t]{3}{*}{$\mathrm{C} 33$} & $\mathrm{C} 1^{\prime}$ & $1.285 \pm 0.030$ & $75.47 \pm 2.20$ & \\
\hline & $\mathrm{C} 5^{\mathrm{b}}$ & $1.691 \pm 0.048$ & $96.24 \pm 3.50$ & \\
\hline & $\mathrm{C} 6$ & $1.695 \pm 0.040$ & $110.53 \pm 4.86$ & \\
\hline G34 & $\mathrm{C} 8$ & $1.079 \pm 0.011$ & $88.36 \pm 1.59$ & $1.256 \pm 0.042$ \\
\hline \multirow[t]{2}{*}{ G36 } & $\mathrm{C} 1^{\prime}$ & $1.163 \pm 0.055$ & $81.59 \pm 4.28$ & \\
\hline & $\mathrm{C} 8$ & $1.094 \pm 0.014$ & $109.05 \pm 2.52$ & $1.301 \pm 0.070$ \\
\hline $\mathrm{C} 37$ & $C 5^{\mathrm{b}}$ & $1.823 \pm 0.040$ & $125.73 \pm 4.12$ & \\
\hline \multirow[t]{2}{*}{$\mathrm{U} 38$} & $\mathrm{C} 5^{\mathrm{b}}$ & $1.770 \pm 0.074$ & $127.35 \pm 8.30$ & \\
\hline & $\mathrm{C} 6$ & $1.732 \pm 0.069$ & $154.79 \pm 14.16$ & \\
\hline \multirow[t]{3}{*}{$\mathrm{C} 39$} & $\mathrm{Cl} 1^{\prime}$ & $1.052 \pm 0.060$ & $95.03 \pm 5.45$ & \\
\hline & $\mathrm{C} 5^{\mathrm{b}}$ & $1.751 \pm 0.043$ & $132.95 \pm 4.82$ & \\
\hline & C6 & $1.527 \pm 0.041$ & $137.62 \pm 7.18$ & \\
\hline \multirow[t]{3}{*}{ U40 } & $\mathrm{C} 1^{\prime}$ & $0.871 \pm 0.087$ & $67.43 \pm 15.27$ & \\
\hline & $\mathrm{C} 5^{\mathrm{b}}$ & $1.671 \pm 0.072$ & $124.20 \pm 7.68$ & \\
\hline & C6 & $1.588 \pm 0.065$ & $119.24 \pm 12.29$ & \\
\hline \multirow[t]{2}{*}{$\mathrm{C} 41$} & $\mathrm{C} 5^{\mathrm{b}}$ & $1.708 \pm 0.042$ & $112.74 \pm 3.95$ & \\
\hline & C6 & $1.662 \pm 0.058$ & $144.12 \pm 10.41$ & \\
\hline \multirow[t]{2}{*}{ U42 } & $\mathrm{C} 5^{\mathrm{b}}$ & $1.535 \pm 0.071$ & $128.99 \pm 8.35$ & \\
\hline & $\mathrm{C} 6$ & $1.615 \pm 0.072$ & $189.94 \pm 24.59$ & \\
\hline G43 & $\mathrm{C} 8$ & $0.898 \pm 0.020$ & $112.66 \pm 3.71$ & $1.187 \pm 0.094$ \\
\hline $\mathrm{C} 44$ & $\mathrm{C} 5^{\mathrm{b}}$ & $1.600 \pm 0.037$ & $118.65 \pm 3.99$ & \\
\hline \multirow[t]{2}{*}{$\mathrm{C} 45$} & $\mathrm{C} 1^{\prime}$ & $1.282 \pm 0.034$ & $68.09 \pm 2.26$ & \\
\hline & $\mathrm{C} 5^{\mathrm{b}}$ & $1.886 \pm 0.054$ & $114.97 \pm 4.56$ & \\
\hline
\end{tabular}


Table S5. $R_{1}, R_{2}$, and C-H NOE measured for E-TAR+ARG using the TROSY-detected (C2 C5 C6 C8) and non-TROSY

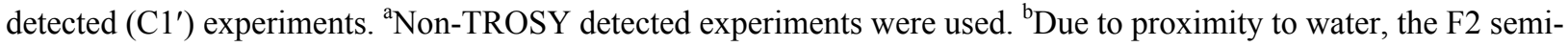

TROSY peak $\left(\Omega_{\mathrm{C} 5}+\pi \mathrm{J}_{\mathrm{CH}}, \Omega_{\mathrm{H} 5}+\pi \mathrm{J}_{\mathrm{CH}}\right)$ was detected.

\begin{tabular}{|c|c|c|c|c|c|}
\hline \multicolumn{2}{|c|}{ Residue } & \multirow{2}{*}{$\begin{array}{c}S_{\mathrm{t}}^{2} \\
0.857 \pm 0.098\end{array}$} & \multirow{2}{*}{$\frac{S_{\mathrm{t}}^{2}(\text { normalized })}{0.857 \pm 0.098}$} & \multirow[t]{2}{*}{$\tau_{\text {eff }}(\mathrm{ps})$} & \multirow{2}{*}{$\frac{\text { Model }}{1}$} \\
\hline A20 & $\mathrm{C} 1^{\prime}$ & & & & \\
\hline G21 & $\mathrm{C} 1^{\prime}$ & $0.958 \pm 0.055$ & $0.958 \pm 0.055$ & & 1 \\
\hline A22 & $\mathrm{C} 1^{\prime}$ & $0.925 \pm 0.053$ & $0.925 \pm 0.053$ & $918.48 \pm 387.48$ & 2 \\
\hline $\mathrm{U} 23$ & $\mathrm{C} 1^{\prime}$ & $0.619 \pm 0.102$ & $0.619 \pm 0.102$ & $280.74 \pm 152.52$ & 2 \\
\hline $\mathrm{U} 25$ & $\mathrm{C} 1^{\prime}$ & $0.622 \pm 0.133$ & $0.622 \pm 0.133$ & $549.65 \pm 430.86$ & 2 \\
\hline A 27 & $\mathrm{C} 1^{\prime}$ & $0.626 \pm 0.072$ & $0.626 \pm 0.072$ & $124.81 \pm 33.38$ & 2 \\
\hline U31 & $\mathrm{C} 1^{\prime}$ & $0.746 \pm 0.102$ & $0.746 \pm 0.102$ & $151.44 \pm 49.83$ & 2 \\
\hline U32 & $\mathrm{C} 1^{\prime}$ & $0.741 \pm 0.106$ & $0.741 \pm 0.106$ & $226.49 \pm 86.46$ & 2 \\
\hline $\mathrm{C} 33$ & $\mathrm{C} 1^{\prime}$ & $0.703 \pm 0.022$ & $0.703 \pm 0.022$ & $131.06 \pm 26.38$ & 2 \\
\hline G34 & $\mathrm{C} 1^{\prime}$ & $0.735 \pm 0.05$ & $0.735 \pm 0.050$ & $201.52 \pm 45.86$ & 2 \\
\hline G36 & $\mathrm{C} 1^{\prime}$ & $0.752 \pm 0.063$ & $0.752 \pm 0.063$ & $142.61 \pm 30.39$ & 2 \\
\hline U38 & $\mathrm{C} 1^{\prime}$ & $0.872 \pm 0.103$ & $0.872 \pm 0.103$ & $181.50 \pm 153.89$ & 2 \\
\hline $\mathrm{C} 39$ & $\mathrm{C} 1^{\prime}$ & $0.852 \pm 0.028$ & $0.852 \pm 0.028$ & $152.40 \pm 58.68$ & 2 \\
\hline U40 & $\mathrm{Cl}^{\prime}$ & $0.823 \pm 0.093$ & $0.823 \pm 0.093$ & $318.30 \pm 195.53$ & 2 \\
\hline U42 & $\mathrm{C} 1^{\prime}$ & $1.000 \pm 0.083$ & $1.000 \pm 0.083$ & & 1 \\
\hline A20 & $\mathrm{C} 2$ & $0.933 \pm 0.025$ & $1.000 \pm 0.025$ & $780.12 \pm 480.27$ & 2 \\
\hline A22 & $\mathrm{C} 2$ & $0.917 \pm 0.017$ & $0.984 \pm 0.017$ & $584.80 \pm 499.65$ & 2 \\
\hline A27 & $\mathrm{C} 2$ & $0.778 \pm 0.077$ & $0.834 \pm 0.077$ & $797.09 \pm 1233.38$ & 5 \\
\hline C19 & $\mathrm{C} 5$ & $0.849 \pm 0.026$ & $0.927 \pm 0.026$ & & 1 \\
\hline U23 & $\mathrm{C} 5$ & $0.699 \pm 0.080$ & $0.763 \pm 0.080$ & $97.43 \pm 43.70$ & 2 \\
\hline $\mathrm{C} 24$ & C5 & $0.405 \pm 0.052$ & $0.442 \pm 0.052$ & $125.42 \pm 10.37$ & 2 \\
\hline $\mathrm{U} 25$ & C5 & $0.535 \pm 0.123$ & $0.584 \pm 0.123$ & $110.57 \pm 30.97$ & 2 \\
\hline $\mathrm{C} 29$ & $\mathrm{C} 5$ & $0.701 \pm 0.036$ & $0.766 \pm 0.036$ & $126.49 \pm 11.76$ & 2 \\
\hline U31 & $\mathrm{C} 5$ & $0.728 \pm 0.064$ & $0.796 \pm 0.064$ & $151.03 \pm 32.79$ & 2 \\
\hline U32 & $\mathrm{C} 5$ & $0.642 \pm 0.023$ & $0.701 \pm 0.023$ & $73.02 \pm 6.53$ & 2 \\
\hline $\mathrm{C} 33$ & $\mathrm{C} 5$ & $0.669 \pm 0.037$ & $0.730 \pm 0.037$ & $104.45 \pm 8.92$ & 2 \\
\hline $\mathrm{C} 37$ & $\mathrm{C} 5$ & $0.684 \pm 0.036$ & $0.747 \pm 0.036$ & $125.96 \pm 10.67$ & 2 \\
\hline U38 & $\mathrm{C} 5$ & $0.767 \pm 0.060$ & $0.837 \pm 0.060$ & $128.16 \pm 29.20$ & 2 \\
\hline $\mathrm{C} 39$ & $\mathrm{C} 5$ & $0.764 \pm 0.036$ & $0.834 \pm 0.036$ & $147.30 \pm 25.23$ & 2 \\
\hline $\mathrm{U} 40$ & $\mathrm{C} 5$ & $0.740 \pm 0.062$ & $0.808 \pm 0.062$ & $130.57 \pm 23.25$ & 2 \\
\hline $\mathrm{C} 41$ & $\mathrm{C} 5$ & $0.876 \pm 0.024$ & $0.957 \pm 0.024$ & & 1 \\
\hline U42 & $\mathrm{C} 5$ & $0.916 \pm 0.030$ & $1.000 \pm 0.030$ & & 1 \\
\hline $\mathrm{C} 44$ & $\mathrm{C} 5$ & $0.884 \pm 0.031$ & $0.966 \pm 0.031$ & & 1 \\
\hline $\mathrm{C} 19$ & $\mathrm{C} 6$ & $0.824 \pm 0.109$ & $0.942 \pm 0.109$ & $70.49 \pm 38.41$ & 2 \\
\hline $\mathrm{U} 23$ & $\mathrm{C} 6$ & $0.733 \pm 0.097$ & $0.837 \pm 0.097$ & $171.73 \pm 58.81$ & 2 \\
\hline $\mathrm{C} 24$ & $\mathrm{C} 6$ & $0.434 \pm 0.071$ & $0.497 \pm 0.071$ & $137.46 \pm 22.57$ & 2 \\
\hline $\mathrm{U} 25$ & $\mathrm{C} 6$ & $0.485 \pm 0.081$ & $0.555 \pm 0.081$ & $155.99 \pm 21.58$ & 2 \\
\hline $\mathrm{C} 29$ & C6 & $0.632 \pm 0.089$ & $0.723 \pm 0.089$ & $127.36 \pm 43.63$ & 2 \\
\hline U31 & C6 & $0.652 \pm 0.108$ & $0.745 \pm 0.108$ & $129.72 \pm 56.95$ & 2 \\
\hline U32 & C6 & $0.503 \pm 0.054$ & $0.575 \pm 0.054$ & $94.27 \pm 19.72$ & 2 \\
\hline $\mathrm{C} 33$ & $\mathrm{C} 6$ & $0.585 \pm 0.071$ & $0.668 \pm 0.071$ & $105.63 \pm 26.15$ & 2 \\
\hline U38 & C6 & $0.657 \pm 0.107$ & $0.751 \pm 0.107$ & $137.78 \pm 71.62$ & 2 \\
\hline $\mathrm{C} 41$ & $\mathrm{C} 6$ & $0.875 \pm 0.109$ & $1.000 \pm 0.109$ & $81.85 \pm 80.48$ & 2 \\
\hline G17 & $\mathrm{C} 8$ & $0.859 \pm 0.020$ & $0.924 \pm 0.020$ & $510.47 \pm 346.37$ & 5 \\
\hline
\end{tabular}




\begin{tabular}{llllll} 
G18 & C8 & $0.930 \pm 0.008$ & $1.000 \pm 0.008$ & $706.59 \pm 288.52$ & 2 \\
A20 & C8 & $0.845 \pm 0.057$ & $0.909 \pm 0.057$ & $553.32 \pm 754.30$ & 5 \\
G21 & C8 & $0.895 \pm 0.027$ & $0.962 \pm 0.027$ & $801.56 \pm 925.83$ & 5 \\
A22 & C8 & $0.889 \pm 0.016$ & $0.956 \pm 0.016$ & $489.49 \pm 194.86$ & 2 \\
G26 & C8 & $0.741 \pm 0.037$ & $0.797 \pm 0.037$ & $853.07 \pm 351.77$ & 5 \\
A27 & C8 & $0.753 \pm 0.037$ & $0.809 \pm 0.037$ & $802.00 \pm 1277.16$ & 5 \\
G28 & C8 & $0.663 \pm 0.017$ & $0.713 \pm 0.017$ & $914.91 \pm 495.18$ & 5 \\
G34 & C8 & $0.524 \pm 0.007$ & $0.564 \pm 0.007$ & $724.30 \pm 165.37$ & 5 \\
G36 & C8 & $0.655 \pm 0.012$ & $0.704 \pm 0.012$ & $745.62 \pm 309.06$ & 5 \\
G43 & C8 & $0.814 \pm 0.026$ & $0.875 \pm 0.026$ & $534.61 \pm 322.41$ & 5 \\
G17 & $\mathrm{N} 1$ & $0.974 \pm 0.005$ & $1.000 \pm 0.005$ & $297.03 \pm 78.05$ & 2 \\
G18 & $\mathrm{N} 1$ & $0.965 \pm 0.017$ & $0.990 \pm 0.017$ & $192.49 \pm 171.81$ & 2 \\
G21 & $\mathrm{N} 1$ & $0.965 \pm 0.010$ & $0.991 \pm 0.010$ & $724.56 \pm 301.81$ & 2 \\
G28 & $\mathrm{N} 1$ & $0.742 \pm 0.016$ & $0.762 \pm 0.016$ & $1443.93 \pm 76.36$ & 5 \\
G34 & $\mathrm{N} 1$ & $0.682 \pm 0.010$ & $0.700 \pm 0.010$ & $1379.83 \pm 47.23$ & 5 \\
G36 & $\mathrm{N} 1$ & $0.705 \pm 0.018$ & $0.724 \pm 0.018$ & $1407.83 \pm 62.56$ & 5 \\
U38 & $\mathrm{N} 3$ & $0.718 \pm 0.051$ & $0.737 \pm 0.051$ & $1490.69 \pm 159.89$ & 5 \\
U42 & $\mathrm{N} 3$ & $0.967 \pm 0.023$ & $0.992 \pm 0.023$ & $152.11 \pm 487.64$ & 2 \\
\hline
\end{tabular}

Table S6. Motional parameters for free E-TAR. $\tau_{\mathrm{eff}}$ is $\tau_{\mathrm{f}}$ for models 1-4 and $\tau_{\mathrm{s}}$ for model 5 .

\begin{tabular}{|c|c|c|c|c|c|}
\hline \multicolumn{2}{|c|}{ Residue } & $S_{\mathrm{t}}^{2}$ & $S^{2}{ }_{t}$ normalized $)$ & $\tau_{\text {eff }}(\mathrm{ps})$ & Model \\
\hline G21 & $\mathrm{C}^{\prime \prime}$ & $0.821 \pm 0.044$ & $0.824 \pm 0.044$ & & 1 \\
\hline $\mathrm{U} 23$ & $\mathrm{C} 1^{\prime}$ & $0.685 \pm 0.130$ & $0.688 \pm 0.130$ & $130.48 \pm 30.44$ & 2 \\
\hline $\mathrm{C} 24$ & $\mathrm{C} 1^{\prime}$ & $0.232 \pm 0.015$ & $0.233 \pm 0.015$ & $162.40 \pm 3.44$ & 2 \\
\hline $\mathrm{U} 25$ & $\mathrm{C} 1^{\prime}$ & $0.433 \pm 0.071$ & $0.434 \pm 0.071$ & $197.97 \pm 36.26$ & 2 \\
\hline U31 & $\mathrm{C}^{\prime}$ & $0.486 \pm 0.141$ & $0.488 \pm 0.141$ & $70.16 \pm 13.32$ & 2 \\
\hline U32 & $\mathrm{C} 1^{\prime}$ & $0.712 \pm 0.117$ & $0.714 \pm 0.117$ & $97.82 \pm 25.37$ & 2 \\
\hline $\mathrm{C} 33$ & $\mathrm{C} 1^{\prime}$ & $0.791 \pm 0.021$ & $0.793 \pm 0.021$ & $175.98 \pm 29.67$ & 2 \\
\hline G36 & $\mathrm{C} 1^{\prime}$ & $0.917 \pm 0.061$ & $0.920 \pm 0.061$ & $80.39 \pm 190.59$ & 2 \\
\hline C39 & $\mathrm{C}^{\prime}{ }^{\prime}$ & $0.996 \pm 0.026$ & $1.000 \pm 0.026$ & & 1 \\
\hline $\mathrm{A} 20$ & $\mathrm{C} 2$ & $0.937 \pm 0.028$ & $1.000 \pm 0.028$ & $268.69 \pm 272.14$ & 2 \\
\hline A22 & $\mathrm{C} 2$ & $0.816 \pm 0.055$ & $0.871 \pm 0.055$ & $1054.84 \pm 441.58$ & 5 \\
\hline A27 & $\mathrm{C} 2$ & $0.930 \pm 0.042$ & $0.993 \pm 0.042$ & $402.04 \pm 384.14$ & 2 \\
\hline C19 & $\mathrm{C} 5$ & $0.893 \pm 0.021$ & $0.950 \pm 0.021$ & & 1 \\
\hline $\mathrm{U} 23$ & $\mathrm{C} 5$ & $0.703 \pm 0.040$ & $0.748 \pm 0.040$ & $82.52 \pm 15.81$ & 2 \\
\hline $\mathrm{C} 24$ & $\mathrm{C} 5$ & $0.219 \pm 0.021$ & $0.233 \pm 0.021$ & $117.94 \pm 3.39$ & 2 \\
\hline $\mathrm{U} 25$ & $\mathrm{C} 5$ & $0.305 \pm 0.025$ & $0.325 \pm 0.025$ & $107.59 \pm 6.16$ & 2 \\
\hline $\mathrm{C} 29$ & $\mathrm{C} 5$ & $0.865 \pm 0.049$ & $0.921 \pm 0.049$ & $56.86 \pm 20.06$ & 2 \\
\hline U31 & $\mathrm{C} 5$ & $0.895 \pm 0.054$ & $0.953 \pm 0.054$ & $104.78 \pm 53.30$ & 2 \\
\hline U32 & $\mathrm{C} 5$ & $0.648 \pm 0.015$ & $0.690 \pm 0.015$ & $49.93 \pm 4.83$ & 2 \\
\hline $\mathrm{C} 33$ & $\mathrm{C} 5$ & $0.663 \pm 0.039$ & $0.706 \pm 0.039$ & $49.82 \pm 7.67$ & 2 \\
\hline $\mathrm{C} 37$ & $\mathrm{C} 5$ & $0.923 \pm 0.023$ & $0.983 \pm 0.023$ & & 1 \\
\hline U38 & $\mathrm{C} 5$ & $0.940 \pm 0.037$ & $1.000 \pm 0.037$ & $0.000 \pm 28.96$ & 2 \\
\hline C39 & $\mathrm{C} 5$ & $0.901 \pm 0.022$ & $0.959 \pm 0.022$ & & 1 \\
\hline U40 & $\mathrm{C} 5$ & $0.892 \pm 0.031$ & $0.949 \pm 0.031$ & & 1 \\
\hline $\mathrm{C} 41$ & $\mathrm{C} 5$ & $0.795 \pm 0.046$ & $0.846 \pm 0.046$ & $33.89 \pm 17.05$ & 2 \\
\hline U42 & $\mathrm{C} 5$ & $0.807 \pm 0.039$ & $0.859 \pm 0.039$ & & 1 \\
\hline $\mathrm{C} 44$ & $\mathrm{C} 5$ & $0.835 \pm 0.021$ & $0.889 \pm 0.021$ & & 1 \\
\hline $\mathrm{C} 45$ & $\mathrm{C} 5$ & $0.837 \pm 0.050$ & $0.891 \pm 0.050$ & $74.13 \pm 19.16$ & 2 \\
\hline
\end{tabular}




\begin{tabular}{|c|c|c|c|c|c|}
\hline C19 & $\mathrm{C} 6$ & $0.812 \pm 0.104$ & $1.000 \pm 0.104$ & $70.73 \pm 20.62$ & 2 \\
\hline $\mathrm{U} 23$ & C6 & $0.715 \pm 0.054$ & $0.880 \pm 0.054$ & $85.73 \pm 9.57$ & 2 \\
\hline $\mathrm{C} 24$ & C6 & $0.197 \pm 0.027$ & $0.243 \pm 0.027$ & $123.29 \pm 11.59$ & 2 \\
\hline $\mathrm{U} 25$ & C6 & $0.277 \pm 0.021$ & $0.342 \pm 0.021$ & $138.89 \pm 10.52$ & 2 \\
\hline $\mathrm{C} 29$ & C6 & $0.762 \pm 0.098$ & $0.939 \pm 0.098$ & $71.71 \pm 16.75$ & 2 \\
\hline U31 & C6 & $0.766 \pm 0.056$ & $0.943 \pm 0.056$ & $122.68 \pm 27.89$ & 2 \\
\hline U32 & C6 & $0.527 \pm 0.015$ & $0.649 \pm 0.015$ & $85.80 \pm 6.40$ & 2 \\
\hline $\mathrm{C} 33$ & C6 & $0.622 \pm 0.077$ & $0.767 \pm 0.077$ & $86.86 \pm 14.39$ & 2 \\
\hline $\mathrm{C} 39$ & C6 & $0.808 \pm 0.106$ & $0.996 \pm 0.106$ & $1023.75 \pm 353.53$ & 2 \\
\hline U40 & C6 & $0.741 \pm 0.077$ & $0.913 \pm 0.077$ & $74.83 \pm 15.14$ & 2 \\
\hline $\mathrm{C} 41$ & C6 & $0.809 \pm 0.112$ & $0.997 \pm 0.112$ & $80.78 \pm 19.13$ & 2 \\
\hline G17 & $\mathrm{C} 8$ & $0.885 \pm 0.028$ & $0.934 \pm 0.028$ & $692.04 \pm 459.42$ & 5 \\
\hline G18 & $\mathrm{C} 8$ & $0.889 \pm 0.026$ & $0.938 \pm 0.026$ & $750.05 \pm 1510.35$ & 5 \\
\hline A20 & $\mathrm{C} 8$ & $0.947 \pm 0.024$ & $1.000 \pm 0.024$ & $455.94 \pm 240.44$ & 2 \\
\hline G21 & $\mathrm{C} 8$ & $0.831 \pm 0.024$ & $0.878 \pm 0.024$ & $740.51 \pm 399.20$ & 5 \\
\hline A 22 & $\mathrm{C} 8$ & $0.918 \pm 0.017$ & $0.969 \pm 0.017$ & $2583.36 \pm 390.14$ & 2 \\
\hline G26 & $\mathrm{C} 8$ & $0.855 \pm 0.021$ & $0.903 \pm 0.021$ & $676.10 \pm 833.48$ & 5 \\
\hline A 27 & $\mathrm{C} 8$ & $0.903 \pm 0.015$ & $0.953 \pm 0.015$ & $1545.91 \pm 317.94$ & 2 \\
\hline G28 & $\mathrm{C} 8$ & $0.816 \pm 0.023$ & $0.861 \pm 0.023$ & $708.66 \pm 323.91$ & 5 \\
\hline G34 & $\mathrm{C} 8$ & $0.666 \pm 0.011$ & $0.703 \pm 0.011$ & $638.99 \pm 147.75$ & 5 \\
\hline G36 & $\mathrm{C} 8$ & $0.814 \pm 0.016$ & $0.859 \pm 0.016$ & $515.68 \pm 153.56$ & 5 \\
\hline G43 & $\mathrm{C} 8$ & $0.847 \pm 0.024$ & $0.894 \pm 0.024$ & $706.74 \pm 338.63$ & 5 \\
\hline G17 & N1 & $0.942 \pm 0.009$ & $0.960 \pm 0.009$ & $1800.37 \pm 241.13$ & 2 \\
\hline G18 & N1 & $0.936 \pm 0.010$ & $0.954 \pm 0.010$ & $1321.05 \pm 194.59$ & 2 \\
\hline G21 & N1 & $0.912 \pm 0.008$ & $0.930 \pm 0.008$ & $1393.71 \pm 106.75$ & 2 \\
\hline G26 & N1 & $0.898 \pm 0.009$ & $0.916 \pm 0.009$ & $1485.80 \pm 108.16$ & 2 \\
\hline G28 & N1 & $0.908 \pm 0.011$ & $0.926 \pm 0.011$ & $1002.50 \pm 132.57$ & 2 \\
\hline G36 & N1 & $0.888 \pm 0.010$ & $0.906 \pm 0.010$ & $1413.39 \pm 106.36$ & 2 \\
\hline U38 & $\mathrm{N} 3$ & $0.934 \pm 0.063$ & $0.952 \pm 0.063$ & $1130.31 \pm 176.55$ & 2 \\
\hline U42 & $\mathrm{N} 3$ & $0.981 \pm 0.036$ & $1.000 \pm 0.036$ & $59.84 \pm 35.13$ & 2 \\
\hline G43 & N1 & $0.964 \pm 0.006$ & $0.983 \pm 0.006$ & $988.40 \pm 1035.14$ & 2 \\
\hline
\end{tabular}

Table S7. Motional parameters for E-TAR+ARG.

\section{$\underline{\text { Supporting References }}$}

1. Jones, J. A.; Hodgkinson, P.; Barker, A. L.; Hore, P. J. J. Magn. Reson. B 1996, 113, 25-34.

2. Jones, J. A. J. Magn. Reson. 1997, 126, 283-286. 